Forum 2020 · 35:428

https://doi.org/10.1007/s12312-020-00853-5

Online publiziert: 1. Oktober 2020

(c) Springer Medizin Verlag GmbH, ein Teil von

Springer Nature 2020

KREBSVERBAND

BADEN-WÜRTTEMBERG E.V.

\section{Dass es "sie“" in Baden-Württemberg immer noch gibt, ist ein Grund zum Feiern. Ihre immer noch nicht verläss- lich geregelte Finanzierung ist das „Haar in der Suppe" - die Nichtaus- breitung auf Bundesebene ist völlig unverständlich, ja ein echtes Ärger- nis, um nicht zu sagen ein Skandal!}

Frau Prof. Heidemann, Frau WohlandBraun, Herr Prof. Schwarz und ich hatten vor gut 30 Jahren eine Idee: Schwerst krebskranke Menschen müssen die Chance haben, eigenverantwortlich zu entscheiden, ob sie stationär oder in ihrer gewohnten häuslichen Umgebung betreut werden wollen. Dabei muss für sie und ihren Angehörigen die Garantie bestehen, jederzeit, schnell und unbürokratisch wieder stationär aufgenommen zu werden.

Es bot sich an, mit der Organisation die in Baden-Württemberg flächendeckend bestehenden Onkologischen Zentren - Tumorzentren (TZ) und Onkologische Schwerpunkte (OSP) - zu betrauen.

Mein Part als junger Verwaltungsmann und ehrenamtlicher Geschäftsführer des Krebsverbandes Baden-Württemberg war es, die Gesundheitsexperten im Sozialministerium und die Krankenkassenchefs zu überzeugen.

Der Krankenhausexperte im Sozialministerium erkannte sofort die Sinnhaftigkeit unseres Vorhabens, wusste aber in seinem gemütlich ausgestatteten Büro mit Hund - sehr genau, wo finanzierungstechnisch „der Hammer hängt".

Von ihm ermutigt bemühte ich mich um einen Termin beim AOK-Chef. Herr Sing war wie das Sozialministerium grundsätzlich sehr angetan von der Idee. Die Schnittstellenproblematik machte ihm Sorgen: „Wen beauftrage ich mit der Ausarbeitung/Umsetzung des Projekts: Die für die Krankenhäuser zuständige $\mathrm{Ab}$ teilung oder die Experten für die Sicherstellung der ambulanten Versorgung?" Bei

Hubert Seiter

Stuttgart, Deutschland

\title{
„30 Jahre Brückenschwestern“
}

den nachfolgenden Gesprächen mit den Ersatzkassen kam es erst gar nicht zur inhaltlichen Diskussion: „Die Zuständigkeitsfrage ist so wesentlich, sie kann nur auf Bundesebene geklärt/entschieden werden" - ein Klärungsprozess, der offensichtlich bis heute noch nicht abgeschlossen ist.

Lange Rede, kurzer Sinn, als zäher Verwaltungsjurist suchte und fand ich die vorläufige Lösung:

Der Krebsverband sammelt ca. 1 Mio. DM, finanziert damit 3 Modellprojekte in Stuttgart, Tübingen und Ravensburg, lässt diese wissenschaftlich begleiten/evaluieren und überzeugt nach dem - selbstverständlich - erfolgreichen Abschluss so die künftigen Kostenträger.

Der „Rest“ ist schnell erzählt: Die AOK und das Sozialministerium waren mit diesem Vorgehen einverstanden. Sie „übersahen" auch großzügig, dass wir eines der Modelle geöffnet hatten für „schwerstkranke Menschen" mit und ohne Krebs.

Die Robert-Bosch-Stiftung stieg als großzügige Geldgeberin ein, Prof. Schwarz sorgte für eine wissenschaftlich hervorragende Begleitung/Evaluation und der Krebsverband sorgte mit Unterstützung der Deutschen Krebsgesellschaft für bundesweites Interesse.

Wenn Sie jetzt glauben, dass heute, gut 25 Jahre nach Abschluss der - sehr erfolgreichen - Projektphase, die „Brückenpflege" bundesweit etabliert und regelhaft finanziert ist, liegen Sie leider falsch!

Das gegliederte Gesundheits- und Finanzierungssystem in Deutschland, mit seiner preußisch-bürokratisch geprägten Tradition, wusste und weiß sich immer noch zu wehren - von „kleinen, widerborstigen aber erfreulich kundenorientiert aufgestellten Selbstverwaltungen wie die LVA/DRV Baden-Württemberg" mal abgesehen.
Liebe Mitstreiterinnen und Mitstreiter, mir ist es regelrecht peinlich bilanzieren zu müssen, dass es bis heute nicht gelungen ist, der hervorragenden Idee einer „sektorenübergreifenden und unbürokratischen Brückenpflege" zum Wohle schwerstkranker Menschen bundesweit zum Durchbruch zu verhelfen.

Mehr noch, es macht mich wütend, wenn ich daran denke, was parallel dazu in dieser langen Zeit geschah. Unglaublich viel Aufwand, Geld und Motivation wurde und wird verschwendet, um höchst bürokratische, ja kafkaesk anmutende „allgemeine und spezielle Versorgungsmonster" für schwerstkranke Menschen in der letzten Lebensphase zu erfinden.

Im Interesse dieser Menschen und ihrer Angehörigen wünsche ich dem Krebsverband von ganzem Herzen viel Erfolg.

\section{Hubert Seiter im August 2020}

P.S.

Eine zweite „unendliche Geschichte", geschrieben nach demselben sozial-bürokratischen Drehbuch, ist auf dem „besten“ Wege: die "sektorenübergreifende Finanzierung der Krebsberatungsstellen"!

Da dort jedoch noch die Kommunen auf Grund ihrer Daseinsfürsorgepflicht ins Boot gehören, werde ich das Ende dieser Geschichte wohl nicht mehr erleben.

\section{Korrespondenzadresse}

\section{Hubert Seiter}

Kriegerstraße 3, 70191 Stuttgart, Deutschland info@lag-selbsthilfe-bw.de www.lag-selbsthilfe-bw.de 